\title{
ULTRAVIOLET FE II ABSORPTION LINES IN HD 59643
}

\author{
Joel A. Eaton \\ Indiana University \\ Bloominton, IN U.S.A.
}

ABSTRACT. The peculiar red giant HD $59643(\mathrm{C} 6,2)$ has a composite spectrum like a symbiotic star's except for the absence of highly ionized species. UV observations show a highly variable hot continuum with superimposed absorptions of $\mathrm{Fe}$ II and the common emission lines of C IV, Si III], C III], C II], and Mg II. These Fe II absorptions, which are practically ubiquitous in interacting binaries, are an effective diagnostic of turbulence and distribution of scattering gas, even at low resolution.

In general symbiotic stars are long-period binaries consisting of a mass-losing red giant and a small hot companion. Ultraviolet radiation from the companion ionizes the cool giant's wind which then emits like a planetry nebula. Charactistic lines of symbiotics require ionization by a source of $\sim 10^{5} \mathrm{~K}$. There are, however, other interacting binaries with similar physical properties but without the emission of highly ionized species. One of these symbiotic-like objects may be the carbon star HD 59643 (Querci et al. 1986). Optical and UV spectra show a blue continuum, Balmer emission lines, unusually strong chromospheric emission lines $(\mathrm{Mg}$ II, Fe II, O I), very strong C IV $\lambda 1550$, and the intersystem Iines C III] and Si III].

In IUE observations at eight epochs, this star's UV continuum has undergone apparently cyclic variations in strength. Figure 1 shows the strongest UV spectrum of HD 59643. Longward of $1550 \AA$, almost all of the absorption dips are $\mathrm{Fe}$ II. We see, extending from 1550 to about $1725 \mathrm{~A}$, a group of depressions produced by Fe II multiplets UV38-45, UV68, and UV8. Features resembling those seen here are also detected to various degrees in ultraviolet spectra of interacting binaries and symbiotic stars. Some examples are HD 35155 (Ake and Johnson 1987), 5 Ceti (Eaton and Barden 1987), SX Cas (Plavec et al. 1982), RY Gem (Plavec and Dobias 1987), 22 Vul (Ake et al. 1985), and AR Pav (Hutchings et al. 1983).

The bottom panel of Figure 1 gives a theoretical spectrum for a $10,000 \mathrm{~K}$ model atmosphere in which the opacity is primarily scattering by individual lines (a few strong ones of $C I I, S i I I$, and $O I$ and the very many of $\mathrm{Fe}$ II from Kurucz 1981), ionization and excitation are in LTE, and line broadening is by turbulence $(40 \mathrm{~km} / \mathrm{s}$ ) and radiative damping. This approach agrees well with the observations, although simple chromospheric attenuation of a hot star's light would serve just as well if the turbulence and column density were chosen properly. 


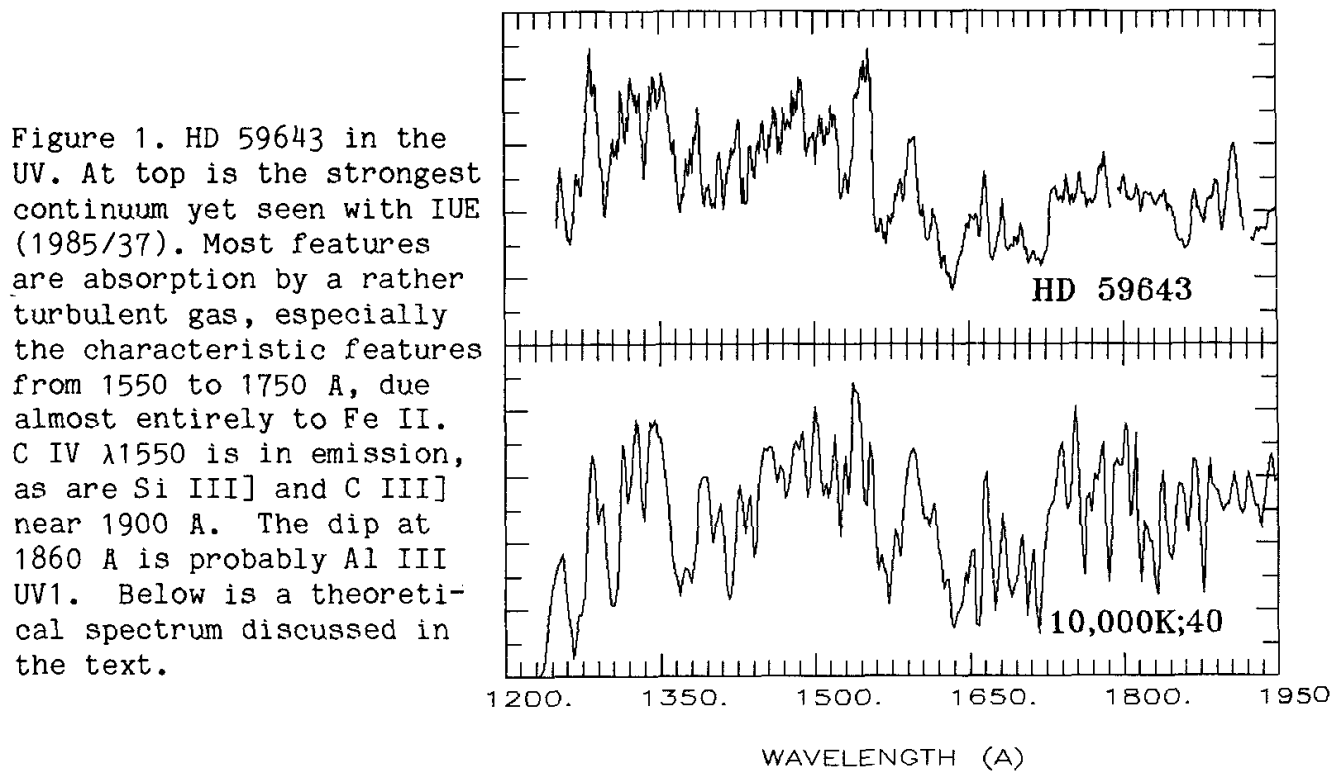

The importance of calculations like these is that they show the crucial role of velocity spread (turbulence?) in determining the strength of UV absorption features. They also suggest a way of studying the distribution of gas about the hot source: If gas is localized in a plane parallel atmosphere or in an isolated cloud along the line of sight, photons will simply be scattered away from us. But if the gas surrounds the star in a detached shell, about as many photons will be scattered into the line of sight as out of it, and the line will be weak (cf. Che et a1. 1983). We may be seeing this effect in the strong Fe II line UV41 $\lambda 1658$ which is much more pronounced in the model than in the observations. Generally, however, the spectrum of HD 59643 seems to be formed by practically pure attenuation. In contrast, 5 Cet shows filling in of the stronger features of $\mathrm{Fe} I I$, such as the dip at $1535 \mathrm{~A}$, from scattering by a chromospheric shell, and AR Pav is surrounded by so much scattering material that it does not seem to eclipse at all in the UV.

\section{REFERENCES}

Ake, T. B., and Johnson, H. R. 1987, Ap. J., in preparation.

Che, A., Hempe, K., and Reimers, D. 1983, Astron. Ap., 126, 225.

Eaton, J. A., and Barden, S. J. 1987, in preparation.

Hutchings, J. B., Cowley, A. P., Ake, T. B., and Imhoff, C. L. 1983, Ap. J., 275, 271 .

Kondo, Y., MCCluskey, G. E., and Parsons, S. B. 1985, Ap. J., $295,580$.

Kurucz, R. L. 1981 , S. A. O. Special Report, No. 390.

Plavec, M. J., and Dobias, J.J. 1987, A.J., 93, 440.

Plavec, M. J., Weiland, J. L., and Koch, K. H. 1982, Ap. J., 256, 206.

Querci, M.\&F., Johnson, H.R., and Baumert, J.H. 1986, Adv.Space Res.,6,215. 